\title{
Reinforcement Learning Methods for Operations Research Applications: The Order Release Problem*
}

\author{
Manuel Schneckenreither ${ }^{[0000-0002-4812-4665]}$ and Stefan \\ Haeussler ${ }^{[0000-0003-2589-1367]}$ \\ Department of Information Systems, Production and Logistics Management, \\ University of Innsbruck, Austria \\ email: manuel.schneckenreither@uibk.ac.at, stef an.haeussler@uibk.ac.at
}

\begin{abstract}
An important goal in Manufacturing Planning and Control systems is to achieve short and predictable flow times, especially where high flexibility in meeting customer demand is required. Besides achieving short flow times, one should also maintain high output and due-date performance. One approach to address this problem is the use of an order release mechanism which collects all incoming orders in an order-pool and thereafter determines when to release the orders to the shop-floor. A major disadvantage of traditional order release mechanisms is their inability to consider the nonlinear relationship between resource utilization and flow times which is well known from practice and queuing theory. Therefore, we propose a novel adaptive order release mechanism which utilizes deep reinforcement learning to set release times of the orders and provide several techniques for challenging operations research problems with reinforcement learning. We use a simulation model of a two-stage flow-shop and show that our approach outperforms well-known order release mechanism.
\end{abstract}

Keywords: operations research, production planning, order release, machine learning, reinforcement learning

The final authenticated version is available online at http://dx.doi.org/10.1007/978-3-030-13709-0_46.

\section{Introduction}

Manufacturing planning and control (MPC) systems play an important role in managing flow of material through manufacturing organizations. An important goal in MPC systems are short and predictable flow times, especially in environments where high flexibility in meeting customer demand is required. Due to complexity reduction purposes MPC systems are often hierarchically structured into two levels [8]. The top level (goods flow control) coordinates the production

\footnotetext{
* This research is partly supported by the Aktion D. Swarovski KG (2016) grant.
} 
units that constitute the logistic chain by coordinated releases of production orders and thus sets the targets for the base level (production unit control; for these terms, see [8]). The base level performs detailed scheduling within the production units. The interface between the top level and the base level is order release which is defined as the transfer of the control over the respective work orders from the top to the base level, which are the decision making units within the production departments.

Within the MPC task the setting of planning parameters plays a crucial role. One of the key parameters is the planned lead time which is defined as the planned time that elapses between the release of an order and its completion (hereinafter denoted as lead time). In contrast, the actual time an order takes to make it through the production system is called flow time and is used as a performance measure. Flow times consist of processing, setup, control, transport, and waiting times, whereas the latter is the governing factor. Waiting times are a result from queuing (e.g. jobs queue before and after processing). Queuing in production systems depends heavily on the amount of jobs in the system (WIP). Thus, waiting times are relatively difficult to estimate which makes the setting of favorable lead times so difficult (e.g., [41,47]).

A common approach to set lead times is either to use fixed (also referred to static) lead times which is mostly used in a Material Requirements Planning (MRP) context (e.g., $[29,42,49]$ ) or a workload limit for the resources or the whole system under study (e.g., workload control or CONWIP; see [43] or [39] respectively) where companies and researchers assume that the set lead times or limits are constant and fixed over the planning horizon. Hoyt was the first to criticize the use of fixed lead times by arguing that lead times should be set dynamically in order to react to the dynamic operational characteristics of the production process [14]. However, a solely reactive approach often generates an erratic order release pattern which is generally denoted as the lead time syndrome (LTS; see e.g., [24]). The LTS can be described as a positive feedback loop in which the flow times increase the lead times via lead time updating [17]. As the lead times increase, more orders are released to the system which results in higher inventory levels and thus in higher flow times again which closes the vicious cycle.

Therefore, in order to overcome the LTS one needs an anticipation function that predicts the flow times of the work orders as a function of the order release decisions which leads to a predictive lead time management approach [36]. Within this stream of research the problem of setting lead times is seen as a forecasting problem. This means that one tries to find lead times that best fit the dynamically changing production environment. Enns et al. develop methods for setting dynamically planned lead times which are based on exponentially smoothed feedback on the flow times of each order at each stage [10]. Similarly, Selcuk et al. [37] apply exponentially smoothed lead times to a capacitated multistage make-to-stock system. However, the latter study shows that exponentially smoothed lead times may induce the LTS in the case of demand variations and high utilization levels. Therefore, a suitable predictive order release model has 
to forecast the future performance, identify pathological behavior and then generate necessary corrective actions to prevent the incurrence of the LTS.

Among a wide variety of prediction methods, machine learning algorithms (e.g., artificial neural networks) are considered the most effective because of their flexible non-linear and interaction effects modeling capability, and consequently have been recommended as a decision support tool for other production planning problems (e.g., due date assignment; see e.g., [15] or [3,48] for a literature review on this topic). To the best of our knowledge, there exist only two papers that use machine learning for making order release decisions [19,31]. However, [19] only decides on the sequence of the releases and [31] limit themselves to a very simple production system. Furthermore, both use a continuous order release method (CONWIP) although in practice order release decisions often need to be made on a daily basis (see [11]).

Therefore, we contribute to this stream of research by developing a periodic adaptive order release mechanism using reinforcement learning which dynamically decides on whether to release an order based on a deep reinforcement learning algorithm. The viability of the developed approach is tested on a multi product, two-stage hypothetical flow shop and is compared to the performance of conventional order release mechanisms. The tested production system setup is characteristic for problems in the operations research domain in the sense of periodic and discrete decisions with the aim to maximize profits and a high degree of complexity. Therefore, the presented reinforcement learning methods can be easily adapted and applied to various problems in operations research.

Clearly, several properties of the production system under investigation (e.g., the work load, utilization, etc.) are to a large extend determined by the used order release mechanism. This means that one experiences 'sampling issues' since the state of the system depends on the order release quantities and thus a training set which is based on a certain order release mechanism is distorted. This makes supervised learning techniques unsuited, since they use the generated data to infer its knowledge on how to release orders and will always follow the characteristics of the chosen release technique for data generation. Therefore, reinforcement learning is the appropriate AI technique to use, since it bases each learning step on its current knowledge and thus is able to explore the whole solution space. Additionally, reinforcement learning takes into account queuing effects which influence system states over multiple periods, since it is designed to connect the output of consecutive periods whereas standard supervised learning techniques would not take care of these interrelations over multiple periods.

The rest of the paper is structured as follows. The next section reviews the relevant literature and thereafter in Section 3 we describe the used methodology. In Section 4 we present the results and conclude our findings in Section 5.

\section{Literature review}

This section is structured into two related fields of research which are the basis for our paper. First, there exist a large body of literature on order release mod- 
els which constitutes the foundation of this paper. Secondly, studies that use techniques based on AI in the field of operations research with a special focus on production planning.

The literature on order release models can be divided into two main streams, namely the conventional rule based order release mechanisms (e.g., $[6,13,47])$ and multi-period optimization models. Note that a review of optimization based order release models is out of the scope of this paper (see [30] for a review on this topic). The literature on rule based order release mechanisms can be divided into two groups: continuous methods, which may trigger a release at any moment in time; and, periodic release methods, for which release decisions take place at the start of each period. This paper focuses on the latter approach. Several rule based order release mechanisms have been proposed over the last decades ranging from very simple (e.g., immediate release) to more sophisticated ones (e.g., backward infinite loading). The backward infinite loading (BIL) technique was introduced by Ackerman in [1] and calculates the release date (RD) of an order $j$ by subtracting its lead time (LT) of its due date (DD):

$$
R D_{j}=D D_{j}-L T_{j}
$$

Besides the two above mentioned uncapacitated approaches also capacitated mechanism were developed. The two most prominent methods are the probabilistic and atemporal approach. The probabilistic approach was introduced by Bechte [5], and is known as Load Oriented Manufacturing Control (LOMC) concept (see also [47]). This order release mechanism estimates the input from jobs upstream to the direct load of a work centre using a depreciation factor based on historical data. The atemporal approach (also called LUMS method) was introduced in [7] and [12] and simply adds direct and indirect load (so called aggregate load).

We divide the second literature stream into three categories:

1. Application of AI techniques to scheduling problems,

2. Application of AI techniques to support order release decisions,

3. Application of AI techniques to make decisions on the order entry level (order inquiry, due date assignment).

Within the first category, several different AI techniques are used to address scheduling problems (for a review see [3]) ranging from expert systems (e.g., [35]) to decision trees (e.g., [26]) or methods using ANNs or ANNs in conjunction with other methods (e.g., [20,34]; see [2] for a recent literature review on scheduling with ANNs).

To the best of the authors knowledge there are only two studies that belong to the second category (e.g., [19,31]). [19] combines a genetic algorithm and an induced decision tree. They test their approach on a small and a large job shop (with three and seven machines respectively) and use a CONWIP release rule to determine when to release an order and use the decision tree to find the sequence for order release and a genetic algorithm to find a sequence for dispatching at each machine. [31] uses a reinforcement learning method for order release in a 
single product, serial flow line and compares its performance (WIP costs) with conventional order release policies (e.g., Kanban and CONWIP). Both use a continuous order release method (CONWIP) although in practice order release decisions often need to be made on a daily basis (see [11]).

The third category of literature in this review uses AI techniques to make decisions on the order entry level. Here, ANN or ANN in combination with genetic algorithms are used to predict flow times in order to set due dates (e.g., $[15,16,20,32,33])$.

As described in the literature review above, there is a lack of studies that use AI techniques for making order release decisions. This is quite interesting since early studies (e.g., [25]) state that dispatching becomes less important when combined with an appropriate order release mechanism. Therefore, we develop a periodic order release model based on deep reinforcement learning and show its viability on a multi-product, two-stage hypothetical flow shop.

\section{Methodology}

This section describes the simulation model, the used conventional order release methods, and the reinforcement learning algorithm including parameter setup and tested variants.

\subsection{Simulation model}

To ensure generalizability, we use a hypothetical flow shop make-to-order manufacturing system similar to the system analyzed in [19]. The simulation model consists of three work centres; each consists of a single machine and can process only one order at a time. The number of orders arriving is uniformly distributed between 3 and 15 order per period and thus with a mean of 9 order per period. In other words, every 106.67 minutes one order arrives in the order pool. Incoming orders are queued at the order pool until released. The due date slack (dds), that is the periods until incoming orders are due, is set to 7. Once released they are queued at each work center and wait until being processed by the machine. The queuing priority is first-come-first-serve. No preemptions are allowed. Order routings are deterministic and embrace two production stages with diverging shape and no return visits. This results in 2 different products. The operation times of the work centres are uniformly distributed, cf. Table 1 . These characteristics lead to a utilization rate of $90 \%$ for the bottleneck work center (WC3) in steady state. Planning periods were set to 960 minutes (16 hours).

To evaluate the performance of the different order release models we define following performance measures similar to literature (e.g., $[4,44])$ :

- Cost related measures: average total holding costs for WIP and finished goods inventory, costs for backorders. There are no earnings, thus the algorithms minimizes the costs. The setup for the actual values of the costs is given in Table 1 and shows that late deliveries are especially expensive. All 


\begin{tabular}{|l||c|c|c|}
\hline Work Center & WC1 & WC2 & WC3 \\
\hline Operation time & $\mathcal{U}(70,130)$ & $\mathcal{U}(130,170)$ & $\mathcal{U}(180,200)$ \\
\hline
\end{tabular}

\begin{tabular}{|l||c|c|c|}
\hline Cost & Wc p. Order/Period & Fc p. Order/Period & Bc p. Order/Period \\
\hline Value & 3 & 10 & 20 \\
\hline
\end{tabular}

Table 1: Operation times (upper half) and costs setup (in monetary unit).

costs are per order and period, and are measured and reported at the end of each period.

- Delivery related measures: mean tardiness of late orders (TA), standard deviation of lateness $(\sigma \mathrm{TA})$.

- Flow time related measures: mean shop floor throughput time (time duration from release until entry of finished goods inventory; SFTT).

The length of each simulation run to evaluate the performance was 1750 periods including a warm-up period of 750 periods. Welch's procedure was applied to approximate the length of the warm-up period (see [18]). Each order release method was evaluated on 25 predefined demand streams.

\subsection{Conventional Order Release Rules}

As external benchmark for comparison we use different parameterized backward infinite loading (BIL) techniques, cf. Equation 1. Note that we set lead times for each product type and not for every order as done by Ackerman [1].

\subsection{Reinforcement Learning Order Release Algorithm.}

Q-Learning in a nutshell. We use the ideas of the Q-Learning agent as introduced in [40] to set lead times $L T_{p}$ for each product type $p$. Furthermore, inspired by $[27,28,38]$ the algorithm is extended and adapted, as well as lifted to an actor-critic model. The action-value function $Q(s, a)$ (also known as $Q$-function) for a state $s \in \mathcal{S}$ and an action $a \in \mathcal{A}$, as well as the policy $\pi$, are represented by feed-forward artificial neural networks, where $\mathcal{S}$ is the set of all states and $\mathcal{A}$ the set of available actions. Furthermore, we use a target network parameters $\theta_{Q}^{T}$ and $\theta_{\pi}^{T}$ which are softly updated using the worker networks $\theta_{Q}$ and $\theta_{\pi}$ respectively as in [21].

In a nutshell the Q-Learning algorithms work as follows. An agent explores the environment by consecutively taking an action $a \in \mathcal{A}$ and observing rewards $r$ as it traverses from one state $s \in \mathcal{S}$ to another environmental state $s^{\prime} \in \mathcal{S}$. According to the observed reward $r$ and future state $s^{\prime}$ the state-action tuple $(s, a)$ is assessed. This value is then stored in the $Q$-function before repeating the process. Nonetheless, Q-Learning assumes an underlying Markov Decision Processes (MDP). Informally, that is a memory-less task with states, actions, rewards and transition probabilities. For a formal definition see for instance [40, p.61ff]. Under a certain policy $\pi=P(a \mid s)$ the optimal action-value function for an observed state $s$ and action $a$ is given by $Q^{\star}(s, a)=\mathbb{E}_{\pi}\left[\sum_{i=0}^{\infty} \gamma^{i} \cdot r_{t+i} \mid s_{t}=\right.$ 
$\left.s, a_{t}=a\right]$ which is the expected sum of rewards $r_{t}$ discounted by $\gamma$ for each time-step $t$ [40]. Q-learning approximates the optimal action-value function by iteratively updating the state-action values while exploring the solution space.

Convergence and Actor-Critic Model. Even though shown to converge to an optimal action-value function $Q^{\star}(s, a)$ if the $Q$-function is represented discretely [46] for most applications a tabular representation is infeasible due to the exponential growth of the memory requirement. Thus, often (deep) ANNs are used in lieu of actual tables to approximate the $Q$-function, which however, results in the fact that the algorithm is unstable or may even diverge [45]. Inspired by $[22,28]$ we partly overcome this issue by using experience replay, which randomly re-trains a batch out of already visited state-action pairs. Therefore, our algorithm stores a set of experiences $e_{t}=\left(s_{t}, a_{t}, r_{t}, s_{t+1}\right)$ and re-trains the $Q$-function of a randomly picked subset of predetermined size of experiences on each time step $t$. Adding to this we additionally extend the algorithm as in A3C [27] to simultaneously learn a policy. Thus we lift the algorithm to be actor-critic and therefore a second neural network $\theta_{\pi}$ is added.

Markov Decision Process. The underlying MDP is unichain and looks as follows. Both, state space $\mathcal{S}$ and action space $\mathcal{A}$ are discrete. Any state $s \in \mathcal{S}$ of the state space is composed of the following information for each product $p$ :

- The currently set lead time $L T_{p} \in\{1,2, \ldots$, dds $\}$ (Recall: Due Date PeriodLead Time $=$ Release Period). Note that we bound the maximum lead time with due date slack dds, which is 7 in our setup.

- Counters $O P_{p, d} \in \mathbb{N}$ for the number of orders in the order pool divided in time buckets with $d \in\{1,2, \ldots$,dds $\}$, which stands for the number of periods until the due date.

- Counters $Q_{i} \in \mathbb{N}$ standing for the number of orders for each queue $i$.

- Counters $F G I_{p, d} \in \mathbb{N}$ of orders in the finished goods inventory divided in time buckets with $d \in\{-5,-4,-3, \ldots$, dds $\}$, which stands for the number of periods until the due date. Orders with a due date with more than 5 periods ago are listed in the counter $F G I_{-5}$.

- Counters $S_{p, d} \in \mathbb{N}$ of shipped orders from the last period divided in time buckets with $d \in\{-5,-4,-3, \ldots$,dds $\}$, which stands for the number of periods until the due date. Orders with a due date with more than 5 periods ago are listed in the counter $S_{-5}$.

The algorithm implicitly learns a function which maps the current state of the production system to a release decision. In an optimal situation it does so by multiply exploring every action for each state and assessing its economic viability.

Recall that orders are released once the due date is within the interval $[t, t+$ lead time], whereas $t$ is the current period. Clearly, this yields bulk releases (either all or no orders with the same due date and product type are released).

The actions space is composed of two independent decisions. These are the relative changes of the lead times to the currently set lead times $L T_{p} \in$ $\{1,2, \ldots$, dds $\}$ for each product $p$. Recall that $p=2$. Furthermore, we restrict 
the action space for each state $s_{t+1}$ according to the last set lead time $L T_{p}$ from state $s_{t}$ by restricting the change of the lead time for consecutive periods to a maximum of 1 . Thus, if $L T_{p}$ is the current lead time for product $p$ the action space for this product is given by $\{1,2, \ldots$, dds $\} \cap\left\{L T_{p}-1, L T_{p}, L T_{p}+1\right\}$. Put differently the algorithm can increase or decrease the lead time by 1 or leave it as it is, as long as it acts within the discrete action space given by the set $\{1,2, \ldots$, dds $\}$ for each type of product. Thus the action space over all products is given by the full enumeration of available actions of the individual products.

Reward. In each period the agent chooses an action which generates a reward while traversing to the next period by simulating the production system. The rewards are the accumulated costs at the end of the period. These costs are consist of the number of backorders, the current WIP level and the number of orders in the inventory. The costs are divided by the normalizing parameter $\eta$ (see Table 2) and then clipped (cut off) to the interval $[-0.5,0.5]$.

Neural Network Setup. After manually testing various depths and layer widths we found two three layer fully-connected networks to be the most satisfying setup of which both have the same shape. The number of nodes and activation functions are 41-ReLU ${ }^{1}-89-\operatorname{ReLU}-20-\operatorname{ReLU}-9$, with the output activations being Softmax (normalized exponential function) for the policy networks $\theta_{\pi}, \theta_{\pi}^{T}$ and Tanh (hyperbolic tangent function) for the Q-networks $\theta_{Q}, \theta_{Q}^{T}$. The output for both networks consist of $3^{2}=9$ nodes. This is due to the fact that all combinations of increase, decrease and no change of the lead time for each product type have to be represented. As expected the policy network outputs 9 percentages for any given state which specify action probabilities. The Q-network output is a value representing the expected discounted (and average) reward.

Adapted Algorithm. Algorithm 1 shows the adapted Q-Learner algorithm. The experience replay memory is filled by executing random actions before the learning process starts. Note that we do not learn state values as done by $\mathrm{A} 3 \mathrm{C}$ but rather state-action values like in Q-Learning, as we use the average of these values for the policy loss (Line 12). Nonetheless we also use a measure of entropy, in particular the Gini-impurity, with parameter $\beta=0.03$ for preventing early convergence (see [27] for implementation details). The network $\theta_{\pi}^{T}$ represents the target policy network, whereas the network $\theta_{Q}^{T}$ represents the target Q-function. All workers operate on the worker networks $\theta_{\pi}$ and $\theta_{Q}$. The binary variable alg is used to determine the used algorithm. In case of alg $=1$ the average reward value is subtracted for the policy loss, while in case of alg $=0$ average reinforcement learning techniques as presented in the algorithm R-learning [23] are used. The idea in average reinforcement learning is that only the biases of the average reward are used as Q-values.

Although the task of setting the lead times for the orders is done periodically there are no episodes. Further, we observed that standard reinforcement learning

\footnotetext{
${ }^{1}$ ReLU stands for rectified linear unit.
} 


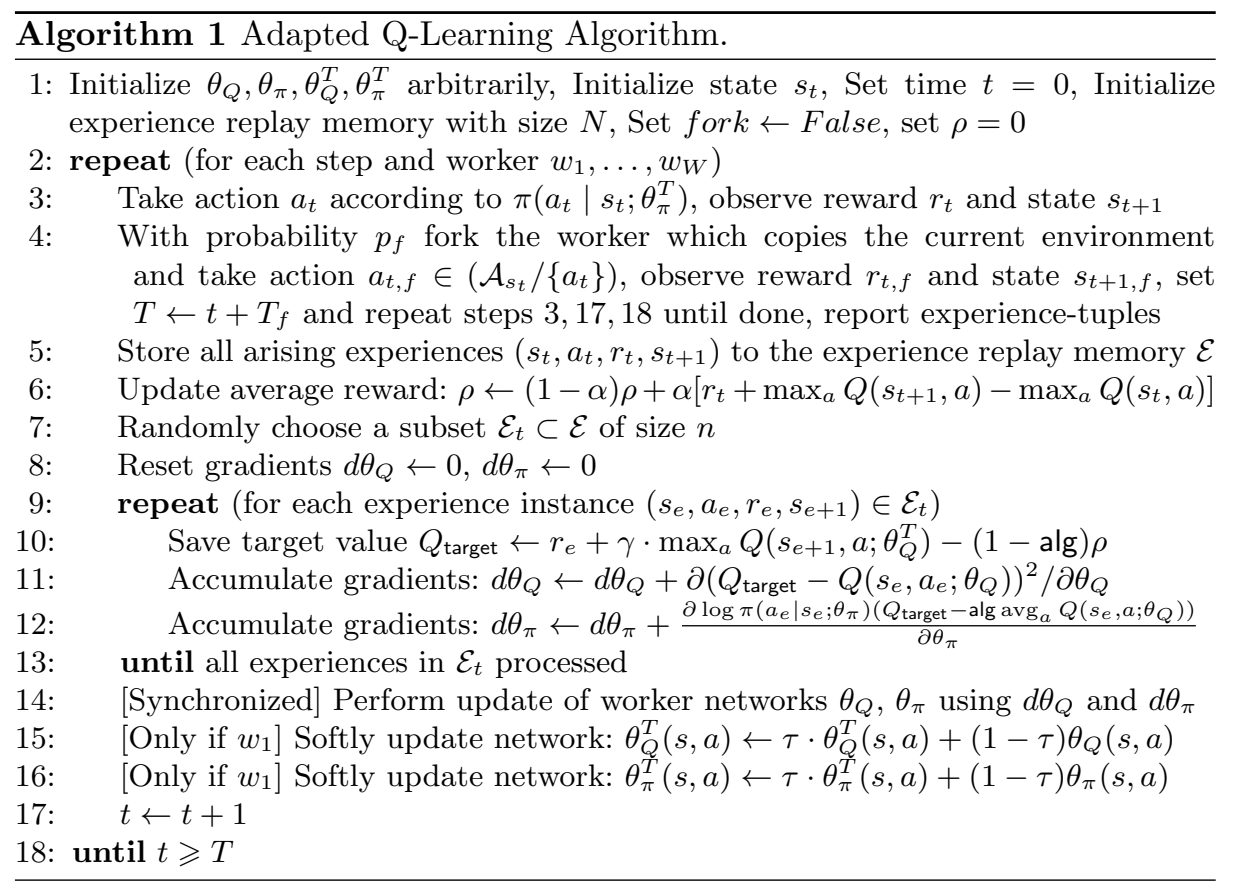

algorithms have problems when presented with rewards in every step, as it is the case in our application as every period the occurring costs are shown to the agent. For standard algorithms this results in ongoing increasing Q-values, until all state-actions values are close to the maximum value. We tackle this by using the average state-value (over all actions) as base for the policy error.

Forking. We have added, so called, forks which are used to search the solution space by copying the current environment of the agent. Thus, at each state the agent explores another randomly chosen action with probability $p_{f}$ (Line 4 ). That means, it copies the current state and takes actions until the $T_{f}$ periods have been observed. All experiences are then returned to the origin worker, which saves them to the experience replay memory. This allows additionally exploring the solution space. We observed a decrease in learning time and an increase in stability of the algorithm with this technique.

Workers. The main worker $w_{1}$, which is the one that is used to evaluate the policy, operates on the learned policy $\pi$. The other 20 workers choose a random action with $20 \%$ probability and otherwise select the actions according to the learned policy $\pi$. Using additionally workers with different or even randomly changing BIL-policies did not yield better results.

Variants. We experiment with the way we represent actual rewards to the algorithm. First we use the standard way which presents the actual costs occurring in one period to the algorithm (MLShipped). However, this results in the fact that the algorithm has to learn two delays. On one hand the standard delay on 


\begin{tabular}{|l||l|c|}
\hline Variant & Description & $\eta$ \\
\hline MLShipped & $\begin{array}{l}\text { Rewards are actual costs of the shipped orders ob- } \\
\text { served at the end of the period. }\end{array}$ & 2500.00 \\
\hline MLShippedAvg & $\begin{array}{l}\text { Same as MLShipped but the rewards are averaged } \\
\text { over the orders. }\end{array}$ & $\begin{array}{c}2500 / 9= \\
277.78\end{array}$ \\
\hline MLShippedAvg ${ }_{A v g R L}$ & $\begin{array}{l}\text { Same as MLShippedAvg but uses average reinforce- } \\
\text { ment learning, thus alg }=0 \text { and } \gamma=0.5 .\end{array}$ & 277.78 \\
\hline MLRelOrds & $\begin{array}{l}\text { The released orders are used to calculate the costs. } \\
\text { MLOrdPool } \\
\text { Mo orders in the order pool at period } t+1 \text { are used } \\
\text { to sum the costs. Averaged over all orders. }\end{array}$ & 2500.00 \\
\hline
\end{tabular}

Table 2: Variants tested.

\begin{tabular}{|l||c|c|c|c|c|c|c|c|c|}
\hline Parameter & $p_{f}$ & $\gamma$ & $\alpha$ & $\tau$ & $N$ & $B$ & Train Iterations & Learn. Rate & L2 \\
\hline Value & 0.2 & $0.995^{2}$ & 0.95 & 0.001 & 30000 & 128 & 4 & 0.01 & 0.0001 \\
\hline
\end{tabular}

Table 3: Parameter Setup.

what actions to choose to traverse to a good state in which high rewards are expected, but on the other hand it must also learn the delay that these rewards are not observed directly, but rather after several periods when the production system outputs the results for the action taken in that state. Therefore, we added the variations of keeping track of either the released orders (MLRelOrds) or the orders in the order pool (MLOrdPool) for each action and present the resulting tuple of action taken, orders affected and costs to the algorithm once the costs are known. Note that as this only affects the policy this variation does not have an effect on the Markov property.

The variants are summarized in Table 2. The mean number of orders arriving at one period is 9 . The values for the min-max normalization $\eta$ is shown on the right and were picked manually after testing multiple values. If not specified then alg $=1$.

\section{Results}

This section gives an overview of the performance of the algorithm compared to the conventional static lead time setting algorithms.

Parameters. Table 3 gives the parameters used for the $Q$-Learning algorithm and the ANN. The fork probability $p_{f}$ was set to 0.2 and the discount factor

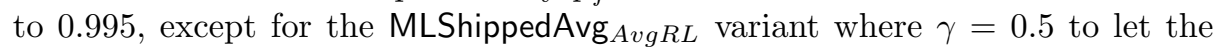
two methods balance out. In each period 128 experiences are retrained out of a set of experiences with size 30000 . The gradients are trained 4 times, with an ANN learning rate of 0.01 and no momentum. The soft update procedure allows this high value for the learning rate, but the momentum was disabled as the networks otherwise diverge. These values were manually optimized beforehand

${ }^{2}$ For MLShippedAvg $\operatorname{AvgRL}_{\text {L }}$ we use $\gamma=0.5$ to allow balancing of average and discounted reinforcement learning. 


\begin{tabular}{|l||c|c|c|c|c|c|c|}
\hline Algorithm/KPI & SUM & BOC & FGIC & WIPC & TARD & $\sigma$ TARD & SFTT \\
\hline MLShippedAvg $_{\text {Avg RL }}$ & 464.84 & 201.47 & 153.49 & 109.89 & 1.80 & 2.57 & 4.35 \\
MLShippedAvg & 473.46 & 326.41 & 63.24 & 83.81 & 1.87 & 2.79 & 3.13 \\
MLOrdPool & $497.83^{\star}$ & 331.57 & 52.18 & 114.08 & 1.92 & 2.84 & 4.23 \\
BIL3 & 535.45 & 400.97 & 28.86 & 105.62 & 2.06 & 2.99 & 4.41 \\
BIL2 & 644.85 & 532.97 & 6.78 & 105.11 & 2.28 & 3.27 & 4.39 \\
MLShipped & 657.85 & 516.27 & 24.13 & 117.45 & 2.33 & 3.33 & 4.84 \\
BIL1 & 803.74 & 698.66 & 0.00 & 105.08 & 2.49 & 3.65 & 4.39 \\
MLRelOrds & 896.51 & 677.89 & 113.38 & 105.24 & 2.46 & 3.64 & 4.21 \\
\hline \multicolumn{7}{|c}{${ }^{\star}$ The p-value of the comparison between MLShippedAvg and MLOrdPool is 0.07364} \\
\hline
\end{tabular}

Table 4: Evaluation results.

by experimenting with multiple setups for all variants. We decided to stick to the values given above as it seems to be the most appropriate setting for the problem. At the beginning the production system is empty, thus a cold start is performed by the agent. The algorithm learned for $150 \mathrm{k}$ periods. The runtime for the evaluations was 1000 periods, with 750 periods startup phase and all evaluations were repeated 25 times.

Results. Table 4 shows the results of the evaluations. The first column denotes the tested order release approaches, namely (i) the static lead time approaches - that calculate the release time of the orders according to (Equation 1) - with different static lead times of 1,2 and 3 (denoted as BIL1, BIL2 and BIL3 respectively) and (ii) the different tested machine learning algorithms (summarized in Table 2) denoted as MLShipped, MLShippedAvg, MLRelOrds, MLOrdPool, and MLShippedAvg $\operatorname{AvgRL}$.

Column two to five show the cost-based performance measures $\left(\cdot 10^{3}\right)$ : the sum over all costs (SUM), backorder costs (BOC), finished goods inventory costs (FGIC) and the costs for held WIP (WIPC). Finally, column six to eight depict the average tardiness of all late orders (denotes as TARD), the standard deviation of tardy orders $(\sigma \mathrm{TARD})$ and the mean shop floor throughput time (SFTT) which is the time an order takes from order release to completion. The measures TARD, $\sigma$ TARD and SFTT are all measured in number of periods.

Table 4 compares the mean of SUM values of all algorithms at a significance level of $p=0.05$ using the Friedman Test [9]. The models shown in grey cells are not statistically distinguishable from each other.

The results in Table 4 show that the standard application of reinforcement learning (MLShipped) is unable to learn the two delays of future rewards. The first one being the standard future reward for taking actions to increase the expected return, whereas the second delay is introduced by the production system which delays the reward according to the current load of the system. Thus the agent would have to learn both delays of rewards, whereas the later one dynamically adapts to the system state. Clearly additional delay of reward opposed by the production system increases the complexity of linking chosen actions to observed 
rewards. This coincides with the suggestion by Zhang to use different neural networks for different lead times [50].

We overcome this problem twofold. On one hand we reduce the complexity the agent has to learn by using future rewards, while on the other hand we provide the agent with the average reward. In case of MLShippedAvg, which only rewards on the average of the shipped orders of the specific period, a continuous output with rather low variance is required for the agent to be able to correctly link the action to the result. To clarify, consider one period with many orders being finished and low average costs to a period with only one order being finished but high costs. In case of MLShippedAvg the agent observes only the average and thus cannot infer the actual average costs over the periods. In our setting however, MLShippedAvg performs well as the output of the production system has low variance.

As can be seen in Table 4, the machine learning algorithms using the average reward (MLShippedAvg $\operatorname{AvgRL}_{\text {, }}$ MLShippedAvg, MLOrdPool) yield the lowest total costs outperforming the conventional static order release models. Furthermore, it is noteworthy that the while the MLShippedAvg $\operatorname{AvgRL}_{\text {yields the lowest lateness }}$ measures (TARD and $\sigma$ TARD) the MLShippedAvg algorithm yields the lowest SFTT. The MLShipped algorithm takes the third last position together with BIL2 and is (next to the MLRelOrds) outperformed by the static lead time approach BIL3.

This shows that the way of representing actual rewards to the machine learning algorithm has a major influence on the performance of the approach. Furthermore, the results show that the average reinforcement learning methods work well in periodic but non-episodic operations research problems, and should be considered a viable research direction.

\section{Conclusions}

This paper adds to the growing body of evidence that machine learning algorithms can contribute positively to a company's performance. The paper describes a successful application of an order release model based on reinforcement learning. The performance is tested on a multi-product, two-stage hypothetical flow shop and is measured by cost/profit, delivery and lead time related measures. We show that our developed machine learning approach outperforms all other tested order release approaches by yielding lower total costs, less mean and standard deviation of tardiness and a shorter shop floor throughput time (SFTT).

The study provides important insights, but we are aware of its limitations. Firstly, the results are limited to the simulated case and the validity of the results for other MTO production systems (e.g., job shop production systems) must be assessed in future studies. Secondly, adding further experimental factors, like machine failures, might contribute in improving the system. It would also be interesting to test the performance of the algorithm to multi-stage production systems and to include scenarios with seasonal demand where one might expect 
even greater benefits from setting the lead times dynamically. Furthermore, a comparison with other order release mechanisms or models (e.g., LUMS/LOMC or optimization based order release models) are an interesting direction for future research.

\section{References}

1. Ackerman, S.: Even-flow a scheduling method for reducing lateness in job shops. Management Technology 3, 20-32 (1963)

2. Akyol, D.E., Bayhan, G.M.: A review on evolution of production scheduling with neural networks. Computers \& Industrial Engineering 53(1), 95 - 122 (2007), http: //www.sciencedirect.com/science/article/pii/S0360835207000666

3. Aytug, H., Bhattacharyya, S., Koehler, G.J., Snowdon, J.L.: A review of machine learning in scheduling. IEEE Transactions on Engineering Management (1994)

4. Baykasoglu, A., Gocken, M.: A simulation based approach to analyse the effects of job release on the performance of a multi-stage job-shop with processing flexibility. International Journal of Production Research 49(2), 585-610 (2011), <GotoISI> : //WOS : 000284413100015

5. Bechte, W.: Theory and practice of load-oriented manufacturing control. The International Journal of Production Research 26(3), 375-395 (1988)

6. Bechte, W.: Load-oriented manufacturing control just-in-time production for job shops. Production Planning \& Control 5(3), 292-307 (1994)

7. Bertrand, J.W.M., Wortmann, J.C.: Production control and information systems for component manufacturing shops. Elsevier Science Inc., New York (1981)

8. Bertrand, J., Wortmann, J., Wijngaard, J.: Production control: a structural and design oriented approach. Elsevier, Amsterdam (1990)

9. Conover, W.: Practical nonparametric statistics. Wiley series in probability and statistics, Wiley, New York, NY [u.a.], 3. ed edn. (1999)

10. Enns, S., Suwanruji, P.: Work load responsive adjustment of planned lead times. Journal of Manufacturing Technology Management 15(1), 90-100 (2004)

11. Gelders, L., Van Wassenhove, L.N.: Hierarchical integration in production planning: Theory and practice. Journal of Operations Management 3(1), 27-35 (1982)

12. Hendry, L., Kingsman, B.: Production planning systems and their applicability to make-to-order companies. European Journal of Operational Research 40(1), 1 - 15 (1989), http://www.sciencedirect.com/science/article/pii/ 037722178990266X

13. Hendry, L., Kingsman, B.: A decision support system for job release in make-toorder companies. International Journal of Operations \& Production Management 11(6), 6-16 (1991)

14. Hoyt, J.: Dynamic lead times that fit today's dynamic planning (quoat lead times). Production and Inventory Management 19(1), 63-71 (1978)

15. Hsu, S.Y., Sha, D.Y.: Due date assignment using artificial neural networks under different shop floor control strategies. International Journal of Production Research 42(9), 1727-1745 (2004), https://doi.org/10.1080/00207540310001624375

16. Karaoglan, A.D., Karademir, O.: Flow time and product cost estimation by using an artificial neural network (ann): A case study for transformer orders. The Engineering Economist 62(3), 272-292 (2017), https://doi.org/10.1080/0013791X. 2016. 1185808 
17. Knollmann, M., Windt, K.: Control-theoretic analysis of the lead time syndrome and its impact on the logistic target achievement. Procedia CIRP 7, 97-102 (2013)

18. Law, A.M., Kelton, W.D.: Simulation Modeling \& Analysis. McGraw-Hill, Inc, New York, 3rd edn. (2000)

19. Lee, C.Y., Piramuthu, S., Tsai, Y.K.: Job shop scheduling with a genetic algorithm and machine learning. International Journal of Production Research 35(4), 11711191 (1997), https://doi.org/10.1080/002075497195605

20. Li, S., Li, Y., Liu, Y., Xu, Y.: A ga-based nn approach for makespan estimation. Applied Mathematics and Computation 185(2), 1003 - 1014 (2007), http://www . sciencedirect.com/science/article/pii/S0096300306008253, special Issue on Intelligent Computing Theory and Methodology

21. Lillicrap, T.P., Hunt, J.J., Pritzel, A., Heess, N., Erez, T., Tassa, Y., Silver, D., Wierstra, D.: Continuous control with deep reinforcement learning. arXiv preprint arXiv:1509.02971 (2015)

22. Lin, L.J.: Reinforcement learning for robots using neural networks. Tech. rep., Carnegie-Mellon Univ Pittsburgh PA School of Computer Science (1993)

23. Mahadevan, S.: Average reward reinforcement learning: Foundations, algorithms, and empirical results. Machine Learning 22(1), 159-195 (Mar 1996), https ://doi. org/10.1007/BF00114727

24. Mather, H., Plossl, G.W.: Priority fixation versus throughput planning. Production and Inventory Management 19, 27-51 (1978)

25. Melnyk, S.A., Ragatz, G.L.: Order review release - research issues and perspectives. International Journal of Production Research 27(7), 1081-1096 (1989), <GotoISI> : //WOS : A1989AC60400003

26. Metan, G., Sabuncuoglu, I., Pierreval, H.: Real time selection of scheduling rules and knowledge extraction via dynamically controlled data mining. International Journal of Production Research 48(23), 6909-6938 (2010), https://doi.org/10. 1080/00207540903307581

27. Mnih, V., Badia, A.P., Mirza, M., Graves, A., Lillicrap, T., Harley, T., Silver, D., Kavukcuoglu, K.: Asynchronous methods for deep reinforcement learning. In: International Conference on Machine Learning. pp. 1928-1937 (2016)

28. Mnih, V., Kavukcuoglu, K., Silver, D., Rusu, A.A., Veness, J., Bellemare, M.G., Graves, A., Riedmiller, M., Fidjeland, A.K., Ostrovski, G., et al.: Human-level control through deep reinforcement learning. Nature 518(7540), 529-533 (2015)

29. Molinder, A.: Joint optimization of lot-sizes, safety stocks and safety lead times in a MRP system. International Journal of Production Research 35(4), 983-994 (1997)

30. Pahl, J., Voß, S., Woodruff, D.L.: Production planning with load dependent lead times: an update of research. Annals of Operations Research 153(1), 297-345 (Sep 2007), https://doi.org/10.1007/s10479-007-0173-5

31. Paternina-Arboleda, C.D., Das, T.K.: Intelligent dynamic control policies for serial production lines. IIE Transactions 33(1), 65-77 (2001), https://doi.org/10. 1080/07408170108936807

32. Patil, R.: Using ensemble and metaheuristics learning principles with artificial neural networks to improve due date prediction performance. International Journal of Production Research 46(21), 6009-6027 (2008)

33. Philipoom, P.R., Rees, L.P., Wiegmann, L.: Using neural networks to determine internally-set due-date assignments for shop scheduling*. Decision Sciences 25(5-6), 825-851 (1994), http://dx.doi.org/10.1111/j.1540-5915.1994.tb01871.x 
34. Raaymakers, W., Weijters, A.: Makespan estimation in batch process industries: A comparison between regression analysis and neural networks. European Journal of Operational Research 145(1), 14 - 30 (2003), http://www. sciencedirect.com/ science/article/pii/S037722170200173X

35. Savell, D.V., Perez, R.A., Koh, S.W.: Scheduling semiconductor wafer production: an expert system implementation. IEEE Expert 4(3), 9-15 (Fall 1989)

36. Schneeweiss, C.: Distributed decision making - a unified approach. European Journal of Operational Research 150(2), 237-252 (2003)

37. Selcuk, B., Fransoo, J.C., De Kok, A.: The effect of updating lead times on the performance of hierarchical planning systems. International Journal of Production Economics 104(2), 427-440 (2006)

38. Silver, D., Hubert, T., Schrittwieser, J., Antonoglou, I., Lai, M., Guez, A., Lanctot, M., Sifre, L., Kumaran, D., Graepel, T., et al.: Mastering chess and shogi by self-play with a general reinforcement learning algorithm. arXiv preprint arXiv:1712.01815 (2017)

39. Spearman, M.L., Woodruff, D.L., Hopp, W.J.: Conwip: a pull alternative to kanban. International Journal of Production Research 28(5), 879-894 (1990), http://www.tandfonline.com/doi/abs/10.1080/00207549008942761

40. Sutton, R.S., Barto, A.G.: Reinforcement learning: An introduction, vol. 1. MIT press Cambridge (1998)

41. Tatsiopoulos, I., Kingsman, B.: Lead time management. European Journal of Operational Research 14(4), 351-358 (1983)

42. Teo, C.C., Bhatnagar, R., Graves, S.C.: An application of master schedule smoothing and planned lead time control. Production and Operations Management 21(2), 211-223 (2012)

43. Thuerer, M., Stevenson, M., Silva, C.: Three decades of workload control research: a systematic review of the literature. International Journal of Production Research 49(23), 6905-6935 (2011)

44. Thuerer, M., Stevenson, M., Silva, C., Land, M.J., Fredendall, L.D.: Workload control and order release: A lean solution for make-to-order companies. Production and Operations Management 21(5), 939-953 (2012)

45. Tsitsiklis, J.N., Van Roy, B.: Analysis of temporal-diffference learning with function approximation. In: Advances in neural information processing systems. pp. 10751081 (1997)

46. Watkins, C.J.C.H., Dayan, P.: Q-learning. Machine Learning 8(3), 279-292 (May 1992), https://doi.org/10.1007/BF00992698

47. Wiendahl, H.: Load-Oriented Manufacturing Control. Springer, Berlin, 1st edn. (1995), http://books.google.at/books-id=e66fmQEACAAJ

48. Wuest, T., Weimer, D., Irgens, C., Thoben, K.D.: Machine learning in manufacturing: advantages, challenges, and applications. Production \& Manufacturing Research 4(1), 23-45 (2016), https://doi.org/10.1080/21693277.2016.1192517

49. Yano, C.: Setting planning lead times in serial production systems with earliness costs. Management Science 33(1), 95-106 (1987)

50. Zhang, G.P.: Avoiding pitfalls in neural network research. IEEE Transactions on Systems, Man, and Cybernetics, Part C (Applications and Reviews) 37(1), 3-16 (2007) 